\title{
An Action-Independent Signature of Perceptual Choice in the Human Brain
}

\author{
Anne E. Urai and Thomas Pfeffer \\ Department of Neurophysiology and Pathophysiology, University Medical Center Hamburg-Eppendorf, 20246 Hamburg, Germany \\ Review of Kelly and O'Connell
}

Imagine walking on a busy street with a large number of people approaching you. To successfully navigate through such a moving crowd and avoid bumping into other pedestrians, you must continuously collect sensory information to decide whether to swerve left or right. But how exactly does your brain transform such sensory information into a meaningful action?

Current sequential sampling models of decision-making posit a decision variable (DV) that reflects accumulated sensory evidence for one alternative over another, and triggers a choice upon reaching one of two bounds (Bogacz et al., 2006). This DV (1) shows a gradual build-up during stimulus viewing, with a slope determined by stimulus strength and (2) reaches a fixed activity level at the decision bound just before response execution. This quantity is encoded in the activity of frontal and parietal oculomotor areas in monkeys (Shadlen and Kiani, 2013), and in the build-up of beta-band power over motor cortex in humans (Donner et al., 2009; O'Connell et al., 2012). Most such electrophysiological markers of evidence accumulation are tightly coupled to motor

Received Feb. 3, 2014; revised Feb. 24, 2014; accepted Feb. $25,2014$.

Anne Urai is supported by the German Academic Exchange Service (DAAD). We thank Tobias Donner, Sander Nieuwenhuis, and Niels Kloosterman for helpful comments on the manuscript.

Correspondence should be addressed to Anne Urai or Thomas Pfeffer, Department of Neurophysiology and Pathophysiology, University Medical Center Hamburg-Eppendorf, Martinistrasse 52, 20246 Hamburg, Germany. E-mail: anne.urai@gmail.com or thms.pfffr@gmail.com.

DOI:10.1523/JNEUROSCI.0477-14.2014

Copyright $\odot 2014$ the authors $\quad 0270-6474 / 14 / 345081-02 \$ 15.00 / 0$ actions used to deliver the response (Donner et al., 2009; Shadlen and Kiani, 2013). This has given rise to the idea that perceptual decision-making is an embodied process, intrinsically linked with its corresponding action. Alternatively, some evidence suggests that neural correlates of the DV may be independent of the motor plan used to indicate the choice (Bennur and Gold, 2011; Filimon et al., 2013).

O'Connell et al. (2012) previously identified such an action-independent DV correlate. Using EEG, they found an eventrelated potential component, the central parietal positivity (CPP), which builds up during stimulus viewing and seems to reflect the integrated evidence provided by those sensory areas encoding the stimulus. Moreover, the signal reaches a fixed level shortly before observers report their choice. Notably, this CPP build-up is independent of the sensory modality of the stimulus and motor requirements of the response. However, the interpretation of these findings was limited by the use of stimuli increasing in strength throughout a trial, which could have led to signal build-up independent of evidence accumulation. In a recent report published in The Journal of Neuroscience, Kelly and O'Connell (2013) showed that the rate of CPP build-up also scales with time-invariant stimulus strength, lending further support for this signal as a neural correlate of sensory evidence accumulation in humans.

Kelly and O'Connell (2013) used a variant of a well studied visual motion discrimination task in which observers judge the net direction of visual motion em- bedded in ongoing dynamic noise. The difficulty of such perceptual decisions can be manipulated by varying the stimulus strength, i.e., the fraction of dots moving in the same direction. In the classical version of this task, stimulus onset evokes strong transients in the EEG signal that are unrelated to the decision process. By displaying a constant stream of $\mathrm{dy}$ namic noise that changes to coherent motion at target onset, Kelly and O'Connell (2013) eliminated these visual transients from the data.

The authors extracted the CPP by averaging the time course of two centroparietal EEG sensors during stimulus viewing, and compared this signal across four levels of stimulus strength. They found that, as predicted, the rate of CPP build-up systematically increased with higher motion strength (Kelly and O'Connell, 2013, their Fig. 1C). Moreover, CPP build-up started to discriminate between different motion strengths approximately $200 \mathrm{~ms}$ after stimulus onset, the same latency as its supposed counterpart in the monkey brain (Kelly and O'Connell, 2013, their Fig. 1C; Shadlen and Kiani, 2013, their Fig. 3C). By splitting the data into six reaction time bins, the authors then showed that CPP build-up predicted observers' reaction times within each level of stimulus strength, as predicted by decision-making models (Kelly and O'Connell, 2013, their Fig. 2). Lastly, CPP build-up preceded the lateralized readiness potential over motor cortex that reflects preparation for a re- 
sponse, suggesting that there is a constant flow of information from regions engaged in the accumulation of sensory evidence to the motor system (Kelly and O'Connell, 2013, their Fig. 1D).Together, these results provide further evidence that the previously identified CPP reflects properties of a neural signature of evidence accumulation as described by mathematical models of decision making.

Having established how stimulus strength influences the dynamics of a decision, the authors investigated the effect of endogenous attentional fluctuations, as indexed by suppression of alpha-band activity (8-12 $\mathrm{Hz}$ ) over visual cortex. The authors showed that lower occipital alpha-band power before target onset resulted in steeper CPP build-up and faster responses. However, since targets could appear at one of three equally likely onset latencies, the expectation that a target will appear (given it has not appeared yet) increased with time. Interestingly, temporal expectation is associated with occipital alpha power suppression and enhances the amplitude of the P3 event-related potential component (Rohenkohl and Nobre, 2011), which is suggested to be highly similar to the CPP (O'Connell et al., 2012). Therefore, the link between ongoing alpha power and CPP build-up could be modulated by the temporal structure of the task.

While it is an interesting possibility that the CPP reflects an accumulating DV, there are other alternatives. In particular, one important point must be taken into consideration when interpreting the CPP signal: it predicts when subjects will respond to a target, but not which specific choice they are about to make (leftwards vs rightwards motion). The CPP thus is not selective about the content of the upcoming choice, in contrast to previously identified signals in human motor (Donner et al., 2009, their Fig 3C) and monkey oculomotor structures (Shadlen and Kiani, 2013, their Fig 3C). Specifically, previous $\mathrm{M} / \mathrm{EEG}$ studies exploited the lateralization of motor activity, which inherently links their findings to the motor action used to indicate the choice (Donner et al., 2009; O'Connell et al., 2012). The CPP's lack of choice-selectivity could result from the recording technique used: due to its limited spatial resolution, EEG picks up the summed postsynaptic activity of a large number of neurons, and could thus reflect activity of two intermixed neuronal populations coding for different motion directions.

Another possibility is that the CPP reflects the observer's confidence in the de- cision. Confidence reflects the certainty of the observer in choosing the correct alternative, and could be reflected in the distance between the DV and the closest decision bound. Importantly, whereas the DV distinguishes between two choice alternatives, a pure confidence signal would be expected to be nonselective with regards to the upcoming choice (Shadlen and Kiani, 2013). One could further distinguish between DV and confidence signals by looking at CPP build-up when observers report a false alarm. It has been suggested that the DV reaches identical levels at response execution for either hits or false alarms, whereas a confidence signal might be expected to have a lower amplitude for false alarms since they could result from noisier sampling (Shadlen and Kiani, 2013). Interestingly, O'Connell et al. (2012, their Fig. 2C) previously reported the latter, lending support to CPP as a confidence signal. To address this possibility, future research could directly probe observers' confidence, for example by using postdecisional wagering (Shadlen and Kiani, 2013), and link this to CPP build-up.

Another, not mutually exclusive, possibility is that CPP build-up reflects phasic activity of the brainstem locus ceruleusnoradrenaline (LC-NE) system, thought to underlie the event-related P3 component of the EEG signal (Nieuwenhuis et al., 2005). Phasic LC-NE activity has been suggested to either reflect a postdecisional process of global gain modulation, triggered by crossing the decision bound (AstonJones and Cohen, 2005), or rather signal task-related information during decision formation (Dayan and Yu, 2006). In line with the latter, a recent report suggests that LC-NE activity, indexed by phasic dilation of the pupil, occurs during the accumulation of sensory evidence (De Gee et al., 2014). While it remains to be tested whether the protracted drive of the pupil system scales with stimulus strength or the efficiency of evidence accumulation, further research could test the hypothesis that phasic LC activity increases neural gain throughout the decision process, thereby boosting the accumulation of evidence, and is reflected in both CPP build-up and pupil dilation.

It has recently been suggested that decision-making processes are not embodied, i.e., perceptual decisions are implemented independent of areas that guide a subsequent action (Bennur and Gold, 2011; Filimon et al., 2013). Kelly and O'Connell (2013) report a neural signal that meets several criteria of a theoretically predicted DV, while additionally being independent of a specific motor response (O'Connell et al., 2012). While future studies need to pinpoint the exact functional significance of the CPP, the report by Kelly and O'Connell (2013) adds to a growing body of work in human electrophysiology (Philiastides et al., 2006; Donner et al., 2009; de Lange et al., 2010) revealing neural dynamics of perceptual decision-making.

\section{References}

Aston-Jones G, Cohen JD (2005) An integrative theory of locus coeruleus-norepinephrine function: adaptive gain and optimal performance. Annu Rev Neurosci 28:403-450. CrossRef Medline

Bennur S, Gold JI (2011) Distinct representations of a perceptual decision and the associated oculomotor plan in the monkey lateral intraparietal area. J Neurosci 31:913-921. CrossRef Medline

Bogacz R, Brown E, Moehlis J, Holmes P, Cohen JD (2006) The physics of optimal decision making: a formal analysis of models of performance in two-alternative forced-choice tasks. Psychol Rev 113:700-765. CrossRef Medline

Dayan P, Yu AJ (2006) Phasic norepinephrine: a neural interrupt signal for unexpected events. Network 17:335-350. CrossRef Medline

De Gee JW, Knapen T, Donner TH (2014) Decision-related pupil dilation reflects upcoming choice and individual bias. Proc Natl Acad Sci U S A 111:E618-E625. CrossRef Medline

de Lange FP, Jensen O, Dehaene S (2010) Accumulation of evidence during sequential decision making: the importance of top-down factors. J Neurosci 30:731-738. CrossRef Medline

Donner TH, Siegel M, Fries P, Engel AK (2009) Buildup of choice-predictive activity in human motor cortex during perceptual decision making. Curr Biol 19:1581-1585. CrossRef Medline

Filimon F, Philiastides MG, Nelson JD, Kloosterman NA, Heekeren HR (2013) How embodied is perceptual decision making? Evidence for separate processing of perceptual and motor decisions. J Neurosci 33:2121-2136. CrossRef Medline

Kelly SP, O'Connell RG (2013) Internal and external influences on the rate of sensory evidence accumulation in the human brain. J Neurosci 33:1943419441. CrossRef Medline

Nieuwenhuis S, Aston-Jones G, Cohen JD (2005) Decision making, the $\mathrm{P} 3$, and the locus coeruleus-norepinephrine system. Psychol Bull 131:510-532. CrossRef Medline

O'Connell RG, Dockree PM, Kelly SP (2012) A supramodal accumulation-to-bound signal that determines perceptual decisions in humans. Nat Neurosci 15:1729-1735. CrossRef Medline

Philiastides MG, Ratcliff R, Sajda P (2006) Neural representation of task difficulty and decision making during perceptual categorization: a timing diagram. JNeurosci 26:8965-8975. CrossRef Medline

Rohenkohl G, Nobre AC (2011) Alpha oscillations related to anticipatory attention follow temporal expectations. J Neurosci 31:1407614084. CrossRef Medline

Shadlen MN, Kiani R (2013) Decision making as a window on cognition. Neuron 80:791-806. CrossRef Medline 